\title{
The flow and heat performance of tree-like network heat sink with diverging-converging channel
}

\author{
Xiugen Zhu ${ }^{1}$, Peng Qian ${ }^{1}$,Zizhen Huang ${ }^{1}$, Chengyuan Luo ${ }^{1}$ And Minghou Liu ${ }^{1, *}$ \\ ${ }^{1}$ Department of Thermal Science and Energy Engineering, University of Science and Technology of China, Hefei 230027, China
}

\begin{abstract}
A tree-like network heat sink with diverging-converging channel is designed, and effect of flow rate, channel diverging-converging angles on the flow and heat dissipation performance of the tree-like network heat sink is analysed and compared by numerical simulation. Results show that the divergingconverging angle of $2^{\circ}$ can reduce the pressure drop by $14 \%$ when inlet mass flow rate is $0.00499 \mathrm{~kg} / \mathrm{s}$. And the maximum temperature, the temperature difference between the maximum and minimum of the heat sink increases by $0.63 \mathrm{~K}$ and $0.92 \mathrm{~K}$ respectively. As the diverging-converging angle increases to $4^{\circ}$, however, it only reduces the pressure drop by $13 \%$ and can not bring more pressure drop due to formation of flow recirculation inside the tree-like network heat sink channel. Therefore, the diverging-converging fractal micro-channel heat sink with $2^{\circ}$ has good heat dissipation performance with obvious lower pumping power.
\end{abstract}

\section{Introduction}

Optimal cooling solutions for thermal management is desperately needed as shrinking the electronic equipment size and increasing the heat generation rate obviously [1]. Tucherman et al. [2] proposed the micro-channel heat sink for these small electronic equipments, and then, many researchers demonstrated its remarkable heat transfer characteristics. In order to improve cooling efficiency of micro-channel heat sink, optimizing geometric design of heat sinks has attracted many researchers' attention. Therefore, tree-like network, a kind of bionic structure existed in nature such as bronchi, breast ductal network and plants, appear and show superior performance on flow and heat transfer.

Many comparisons between the tree-like network heat sink and different traditional structural exchangers were performed. Wang et al. [3] applied 3D printing technology in Manufacturing tree-like network heat exchanger and compared it with traditional spiral-tube exchangers in the performance of heat transfer and flow. Numerically and experimentally, the results showed that the structure of fractal-tree-like can reduce more pressure drop and have a higher value of coefficient of performance. Liu etal. [4] employed tree-like networks in heat-generating discs with point heat sinks and found that these tree-like networks have better heat transfer performance than the classic needle structures. Yu et al. [5] found that the tree-like network micro-channels can improve the characteristic of heat transfer, compared with straight micro-channels. They also proposed that the aspect ratios had great influence on flow and heat transfer. Further, Li et al. [6] proposed geometric optimization method to enhance cooling ability of heat sink. The treelike network they formed was demonstrated to make heat sink faster and more efficient.

In addition, the influence of parameters of tree-like network on thermal and flow efficiency was also presented. Jing and Song [7] investigated the influence of the channel diameter ratio, length ratio, branching level, channel length, branching number on tree-like networks' hydraulic and thermal performance, and two different constraint, surface area constraint and volume constraint, in tree-like networks were compared. The results showed different channel parameters have different influence on different size constraint. Wang et al. [8] studied tree-like structure and proposed that the increase of both number of channel nets and channel levels can decrease the maximum temperature. $\mathrm{Xu}$ et al. [9] established analytical expressions for laminar gas flow resistance in tree-like structure, and found changing diameter scale factor can help improve flow performance. Xu et al. also [10] found that the fractal tree-like multilayer microchannel can enhance heat transfer as the number of layer increases. Liang et al. [11] built tree-like networks with polygonal loops, compared undamaged and damaged structure on the performance of permeability when the structural parameters are different. Miao et al. [12] established a novel model for flow performance in damaged structure. The results showed significant effect of damaged structure compared with normal structure.

Most of above studies were based on circular tube, while the tree-like network based on rectangular section area is different. Jing et al. [13] studied the Murray's law for tree-like rectangular network with constant channel and found Murray's law can't be employed in tree-like

${ }^{*}$ Corresponding author. Tel.: +86055163603127.

E-mail address:mhliu@ustc.edu.cn (Minghou Liu). 
microchannel when the channel is rectangular. Liu et al. [14] proposed a novel tree-like network heat sink, based on rectangular section area, to enhance heat transfer and studied the influence of the inlet width and angle of the oblique channels on the pressure drop. Pulsation flow through tree-like rectangular micro-channel was found to reduce the maximum temperature when the flow is laminar [15]. Chester et al. [16] introduced numerical simulation(RNS) to study turbulence flow over tree-like fractals.

It is obvious that most of the attention on tree-like network was given to uncover physics of fluid flow through microchannel with uniform cross-section, while flowing through non-uniform microchannels has not received sufficient attention. Diverging-converging micro-channels (diamond) are characteristic non-uniform channels and have numerous applications in medicine, engineering andbiology, such as in flow rectification [17], DNA stretching [18],particle separation [19]. Some studies are carried on flow in diverging-converging channels [20-22]. It is observed that large convergencedivergence angle can cause laminar for Reynolds number, $\operatorname{Re}>400$, flowing instability and produced turbulence [23]. The above observations show that the divergingconverging channel is substantially different from straight channels on flow performance. Therefore, studying the effect of geometrical configuration on flow and heat transfer inside the tree-like network structure is of great significance.

In this paper, the diverging-converging channel is introduced into the tree-like network, and its flow and heat transfer performance are studied by numerical simulation, and compared with the uniform cross-section tree-like network. The effect of diverging-converging angles on flow behavior is studied in detail. An appropriate diverging-converging angle is also identified in the literature.

\section{Design consideration}

In this study, a disk heat sink with self-similar tree-like network formed by rectangular channels is designed. The inlet of liquid is on the center of the disc, and the liquid flows into the fractal tree-like network along the channel until it flows out from the last micro-channel, as shown in Figure 1 (a) Compared with the traditional uniform crosssection tree-like network, in this study, the divergingconverging angle is employed in the tree-like network, as shown in Figure 1 (b) The same diverging-converging angle $\alpha$ is added in the middle of each channel level, while other parameters such as length and width of the channel remain constant. (a)

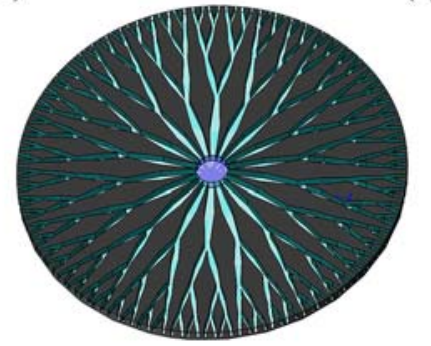

(b)

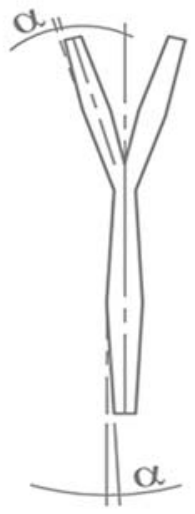

Figure 1. Tree-like network structure with divergingconverging angle.

In present study, $\mathrm{L}_{\mathrm{k}}$ and $\mathrm{W}_{\mathrm{k}}$ are the length and width of rectangular channel at kth branching level respectively, while $\mathrm{H}$ is the height of the channel and keeps constant in each tree-like network. The length ratio and width ratio of the $(\mathrm{k}+1)$ th and $\mathrm{kth}$ branching levels are defined as,

$$
\begin{aligned}
& L_{k+1} / L_{k}=\alpha \\
& W_{k+1} / W_{k}=\beta
\end{aligned}
$$

Therefore, the length and width of the kth branching level can be expressed as,

$$
\begin{aligned}
& L_{k}=L_{0} \alpha^{k} \\
& W_{k}=W_{0} \beta^{k}
\end{aligned}
$$

Where $\mathrm{L}_{0}$ is the length of the initial channel and $\mathrm{W}_{0}$ is the width of the initial channel.

Therefore, the hydraulic diameter of the kth branching level channel can be expressed as,

$$
D_{k}=2 W_{k} H /\left(W_{k}+H\right)=2 W_{0} \beta^{k} H /\left(W_{0} \beta^{k}+H\right)
$$

The hydraulic diameter of the initial channel can be expressed as,

$$
D_{0}=\frac{2 W_{0} H}{\left(W_{0}+H\right)}
$$

\section{Math model}

A three-dimensional tree-like network heat sink with four branch levels is employed, because it has high thermal efficiency and reduces the manufacturing difficulty [24]. The thickness of heat sink is $0.2 \mathrm{~mm}$, the length ratio is 2 $1 / 2$, and the width ratio is $2^{-1 / 3}[25]$. The overall size is displayed as table 1 . 
Table 1. The size of each branching level of tree-like network in heat sink.

\begin{tabular}{llll}
\hline $\begin{array}{l}\text { Branching } \\
\text { level k }\end{array}$ & $\mathbf{H} / \mathbf{m m}$ & $\mathbf{W}_{\mathbf{k}} / \mathbf{m m}$ & $\mathbf{L}_{\mathbf{k}} / \mathbf{m m}$ \\
\hline 1 & 0.5 & 0.35 & 4.4 \\
2 & 0.5 & 0.28 & 3.08 \\
3 & 0.5 & 0.224 & 2.156 \\
4 & 0.5 & 0.1792 & 1.5092 \\
\hline
\end{tabular}

In this study, deionized water is used for laminar forced convection. The heat sink is a micro-channel structure directly etched on copper substrate. The mass flow inlet is adopted and the flow is perpendicular to $\mathrm{Z}$ direction. The bottom surface of the heat sink is the heating surface. In the simulation, the width, height and length of each branching level channel keep constant. Only the diverging-converging angle $\alpha$ is variable, set as $0^{\circ}, 1^{\circ}, 2^{\circ}, 3^{\circ}$, and $4^{\circ}$ respectively. The heat and mass transfer of the tree-like network heat sink can be governed by following equations.

Continuity equation:

$$
\nabla \cdot \vec{v}=0
$$

Momentum equation:

$$
\rho(\vec{v} \cdot \nabla \vec{v})=\vec{f}-\nabla p+\mu \cdot \nabla^{2} \vec{v}
$$

Energy equation of fluid:

$$
\rho c_{p}\left(\vec{v} \cdot \nabla T_{f}\right)=\lambda_{f} \nabla^{2} T_{f}
$$

Where $\mathrm{v}$ is the velocity of the fluid, $\mathrm{f}$ is the gravity of the fluid, $c_{p}$ is the specific heat capacity of the fluid, $T_{f}$ is the temperature of the fluid, $\lambda_{\mathrm{f}}$ is the thermal conductivity of the fluid.

In order to predict the thermal and flow performance of microchannel heat sink more accurately, threedimensional numerical analysis is carried out. Using geometric symmetry, as shown in Figure 2, the calculation domain is simplified to $1 / \mathrm{N}$ of the whole heat sink. For example, there are 18 fractals in this model, $1 / 18$ of the whole heat sink is adopted as the calculation domain including a fractal and adjacent solid area.

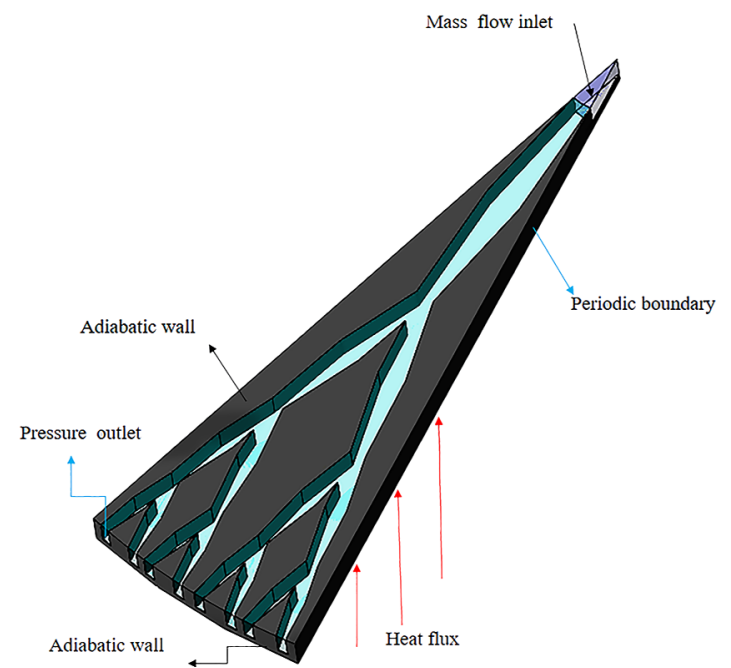

Figure 2. The calculation domain including a fractal and adjacent solid area.

The boundary conditions are as follows:

(1) Inlet: the inlet temperature of the fluid is set at $293 \mathrm{~K}$, and the inlet condition is mass flow inlet, ranging from $0.0025 \mathrm{~kg} / \mathrm{s}$ to $0.0066 \mathrm{~kg} / \mathrm{s}$.

(2) Wall: a constant and uniform heat flow of $50 \mathrm{~W} / \mathrm{cm}^{2}$ is applied to the heated bottom surface. The circular arc side and upper wall are assumed to be adiabatic.

(3) Outlet: the fluid flows out of the last channel freely, and is set as pressure outlet. The physical parameters of the fluid are assumed to be unchanged.

The pumping power that pushes fluid into the treelike network can be expressed as,

$$
\mathrm{W}=\Delta \mathrm{P} \cdot \mathrm{Q}
$$

Where Q is the volume of fluid in the network.

Temperature uniformity is the most important characteristic of the fractal tree-like network heat sink, evaluated by the maximum temperature difference on heated surface of the heat sink.

$$
\Delta T=T_{w_{-} \max }-T_{w_{-} \min }
$$

Where $T_{\mathrm{w} \text { max }}$ is maximum temperature of heated bottom surface, $T_{w_{-} \min }$ is minimum temperature of heated bottom surface.

\section{Simulation model}

The computational fluid dynamics (CFD) simulation tool, fluent, is used to calculate the pressure drop, temperature difference and velocity distribution of heat exchanger. The liquid used in this study is deionized water. The mass flow rate is controlled between $0.0025 \mathrm{~kg} / \mathrm{s}$ and 0.0066 $\mathrm{kg} / \mathrm{s}$. The laminar flow model is selected, and the grid is structured grid. The steady-state and pressure equations are solved numerically using the momentum type. Second order upwind scheme is used for convection and diverging-converging fluxes. Simple algorithm is used 
for pressure velocity coupling. The convergence criterion is that the velocity and continuity residuals of are less than $1 \times 10^{-4}$ for the continuity equation, and the temperature residual is less than $1 \times 10^{-8}$ for the energy equation. In addition, the fluctuation of the wall maximum temperature and the total pressure drop of the tree-like network is less than $0.0005 \%$.

The numerical model is verified by comparing numerical simulation results. Table 2 and table 3 show the comparison in terms of pressure drop and the maximum wall temperature for water cooled minichannel geometry (the channel height is $6 \mathrm{~mm}$, channel width is $0.5 \mathrm{~mm}$, channel thickness is $0.3 \mathrm{~mm}$, and plate thickness is $0.2 \mathrm{~mm}$ ) with velocity between $0.3 \mathrm{~m} / \mathrm{s}$ and $1 \mathrm{~m} / \mathrm{s}$ [26]. The maximum deviation between the present result and results from the literature for $\triangle \mathrm{P}$ and $\mathrm{T}_{\mathrm{w} \text { max }}$ are found to be $1.9 \%$ and $0.3 \%$ respectively. A good agreement is readily seen.

Table 2. Comparison of the total pressure drop with the literature data [26].

\begin{tabular}{llll}
\hline $\mathbf{v}(\mathbf{m} / \mathbf{s})$ & $\begin{array}{l}\triangle \mathbf{P}(\mathbf{P a}) \\
\text { Present }\end{array}$ & $\begin{array}{l}\Delta \mathbf{P}(\mathbf{P a}) \\
\mathbf{R e f}[\mathbf{2 6}]\end{array}$ & $\begin{array}{l}\% \text { diff. in } \\
\Delta \mathbf{P}(\mathbf{P a})\end{array}$ \\
\hline 1 & 1035.16 & 1047.67118 & 1.19419 \\
0.9 & 900.9 & 913.38297 & 1.366674 \\
0.8 & 772.82 & 779.09476 & 0.805391 \\
0.7 & 651.21 & 658.99549 & 1.181418 \\
0.6 & 536.34 & 538.89622 & 0.474344 \\
0.5 & 428.45 & 425.89142 & 0.60076 \\
0.4 & 325.77 & 319.47434 & 1.97063 \\
\hline
\end{tabular}

Table 3. Comparison of the wall maximum temperature with the literature data [26].

\begin{tabular}{lllll}
\hline $\mathbf{v}(\mathbf{m} / \mathbf{s})$ & $\begin{array}{l}\mathbf{T}_{\mathbf{w} \_\max }(\mathbf{K}) \\
\text { Present }\end{array}$ & $\begin{array}{l}\mathbf{T}_{\mathbf{w} \_\max }(\mathbf{K}) \\
\mathbf{R e f}[\mathbf{2 6}]\end{array}$ & $\begin{array}{l}\mathbf{\%} \quad \text { diff. } \\
\mathbf{T}_{\mathbf{w} \_\max }(\mathbf{K})\end{array}$ \\
\hline 1 & 321.89 & 321.18 & 0.220572 & in \\
0.9 & 322.38 & 321.616 & 0.236987 & \\
0.8 & 322.9 & 322.124 & 0.240322 & \\
0.7 & 323.58 & 322.768 & 0.250943 \\
0.6 & 324.38 & 323.444 & 0.28855 \\
0.5 & 325.34 & 324.392 & 0.291387 \\
0.4 & 326.58 & 325.572 & 0.308653 \\
\hline
\end{tabular}

For the grid independence test, three sets of grid $(655560,1403245,2164234)$ are used. The case of the $0^{\circ}$ tree-like network heat sink and the inlet mass flow of $0.0025 \mathrm{~kg} / \mathrm{s}$ is considered. The deviation in the maximum wall temperature and the total pressure drop between 1403245 grid number and 2164234 grid number is less than $0.02 \%$. Therefore, the grid number of 1403245 is selected for calculation.

\section{Results and discussion}

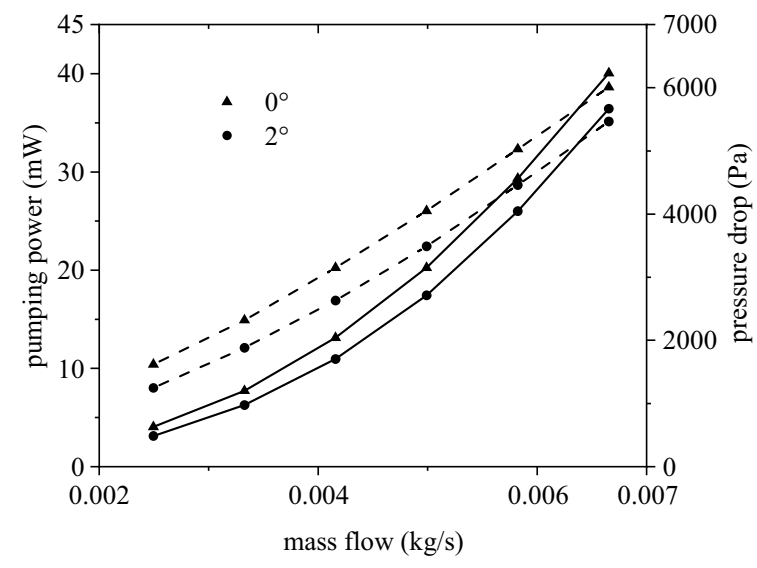

Figure 3. Effect of mass flow rate on pumping power and pressure drop in the tree-like network heat sink with $0^{\circ}$ and $2^{\circ}$ diverging-converging angles.

Figure 3 shows the total pumping power (solid line) and pressure drop (dash line) with mass flow rate for different diverging-converging angle. It is clear that with the increase of mass flow, the total power of tree-like network heat sink increases. However, the total pumping power of tree-like network heat sink with $2^{\circ}$ divergingconverging angle is less than that of tree-like structure with $0^{\circ}$ diverging-converging angle, and is reduced by $22 \% \sim 9 \%$. Moreover, as the mass flow rate increases, the total pumping power of $2^{\circ}$ diverging-converging angle heat sink is reduced more. When mass flow rate is $0.0025 \mathrm{~kg} / \mathrm{s}$, the total pumping power of $2^{\circ}$ divergingconverging angle heat sink is reduced by $0.93 \mathrm{~mW}$. However, when mass flow rate is $0.0066 \mathrm{~kg} / \mathrm{s}$, the total pumping power of $2^{\circ}$ diverging-converging angle heat sink is reduced by $3.6 \mathrm{~mW}$.

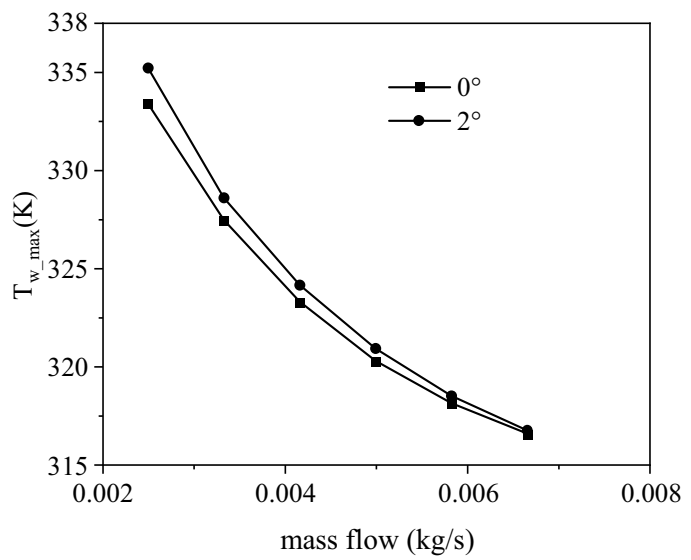

Figure 4. Effect of mass flow rate on the maximum wall temperature for the tree-like network heat sink with $0^{\circ}$ and $2^{\circ}$ diverging-converging angles. 


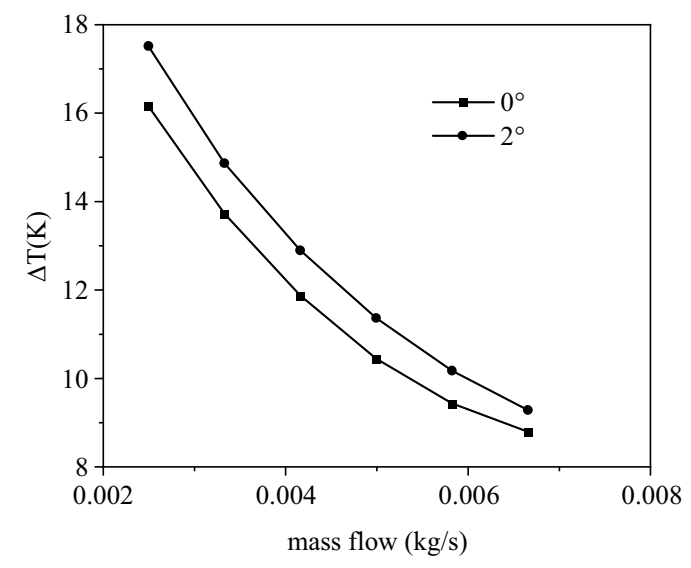

Figure 5. Effect of mass flow rate on the temperature difference for tree-like network heat sink with $0^{\circ}$ and $2^{\circ}$ divergingconverging angles.

The Maximum wall temperature of heat sink reflects the heat dissipation performance. Figure 4 compares the maximum wall temperature of the heat sink with $0^{\circ}$ and $2^{\circ}$ diverging-converging angle. The results show that the maximum wall temperature of heat sink decreases with the increase of flow rate. When the mass flow rate is $0.0025 \mathrm{~kg} / \mathrm{s}$, the maximum wall temperature of heat sink with $0^{\circ}$ diverging-converging angle is $333.40 \mathrm{~K}$, while that of $2^{\circ}$ is $335.23 \mathrm{~K}$, which is $1.83 \mathrm{~K}$ higher than that of $0^{\circ}$. This indicates the increase of the channel expansion angle causes the heat dissipation performance of the heat sink to decrease. However, when the mass flow rate reaches $0.0066 \mathrm{~kg} / \mathrm{s}$, the maximum wall temperature of the heat sink with $0^{\circ}$ and $2^{\circ}$ diverging-converging angle is $316.61 \mathrm{~K}$ and $316.77 \mathrm{~K}$ respectively, and the difference between them is only $0.16 \mathrm{~K}$. This shows that as the flow rate increases, the heat dissipation caused by changes in channel cross-section decreases.

The temperature difference between the maximum and the minimum wall temperature represents the temperature uniformity of the heat sink. Figure 5 compares the variation of maximum and minimum wall temperature difference with mass flow rate between $0^{\circ}$ and $2^{\circ}$ diverging-converging angle of heat sink. When the mass flow rate is $0.0025 \mathrm{~kg} / \mathrm{s}$, the maximum and minimum wall temperature difference of the heat sink with $0^{\circ}$ and $2^{\circ}$ diverging-converging angle is $16.16 \mathrm{~K}$ and $17.52 \mathrm{~K}$. However, when the mass flow rate reaches $0.0066 \mathrm{~kg} / \mathrm{s}$, the wall temperature difference of the heat sink with $0^{\circ}$ and $2^{\circ}$ diverging-converging angle is $8.8 \mathrm{~K}$ and $9.29 \mathrm{~K}$ respectively. It is obvious that, when the mass flow rate increases, the uniformity of wall temperature gets better.

Figure 4 and Figure 5 show that although the maximum wall temperature and wall temperature difference of the heat sink with $2^{\circ}$ diverging-converging angle are higher than those with $0^{\circ}$ diverging-converging angle, the difference between them will gradually decrease with the increase of mass flow rate.Therefore, the tree-like network heat sink with $2^{\circ}$ divergingconverging angle has no obvious influence on the heat dissipation performance and the temperature uniformity of the heat sink.

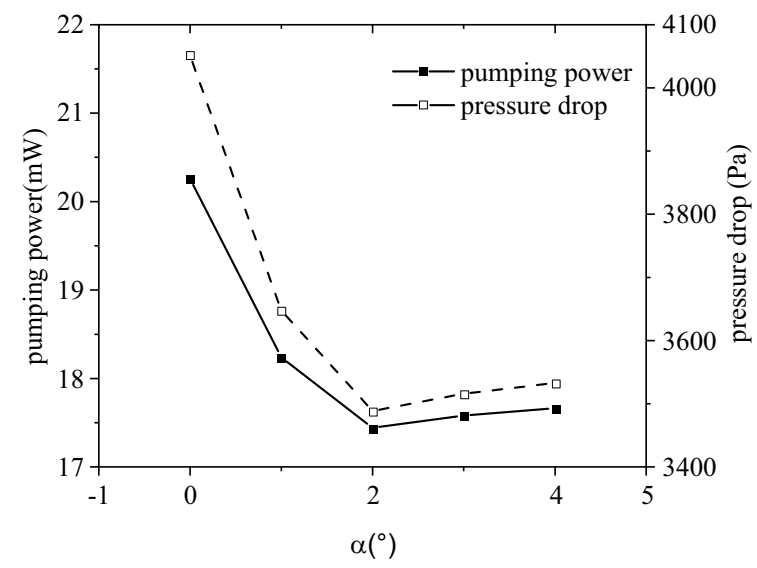

Figure 6. Effect of diverging-converging angle on pumping power and pressure drop of tree-like network heat sink.

Effect of the diverging-converging angle on the total power and total pressure drop when inlet mass flow rate is $0.00499 \mathrm{~kg} / \mathrm{s}$ is presented in Figure 6. It shows that the total power of tree-like network heat sink first decreases, and then increases with the increase of divergingconverging angle. The change of total power is due to the decrease of velocity and inertia force when the hydraulic diameter of the channel increases. Therefore, less pressure drop and less power are required to flow through the channel. However, when the diverging-converging angle reaches $4^{\circ}$, the total power increases, which is due to the increase of pressure loss as the backflow generated by larger diverging-converging angle inside the tree-like channel. And the diverging-converging angle of $2^{\circ}$ and $4^{\circ}$ reduce the pressure drop by $14 \%$ and $13 \%$ respectively. The detailed reasons will be explained below.

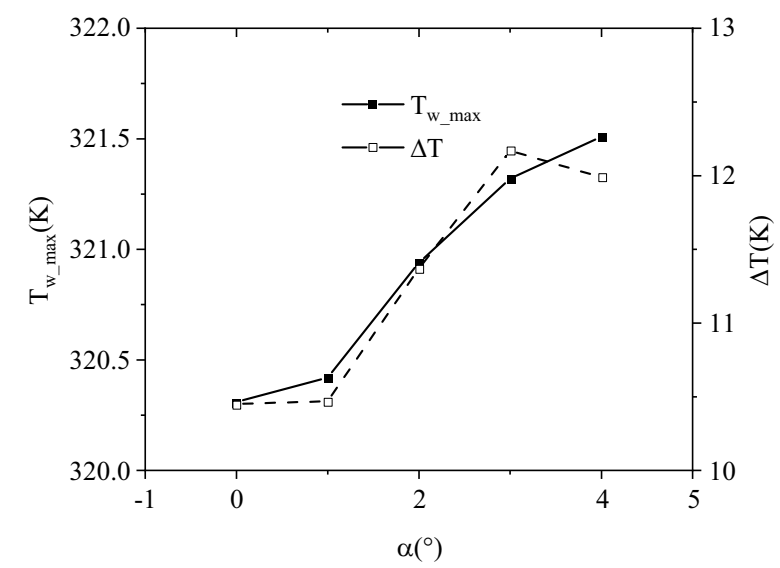

Figure 7. Effect of diverging-converging angle on the maximum temperature and the temperature difference at the bottom of tree-like network heat sink.

Figure 7 shows that the maximum temperature and the temperature difference of the bottom surface of the tree-like network heat sink with different divergingconverging angle when the inlet mass flow rate is $0.00499 \mathrm{~kg} / \mathrm{s}$. The results show that the maximum temperature of the bottom surface of the heat sink increases with the increase of the diverging-converging angle. And it increases by $0.11 \mathrm{~K}$ at $1^{\circ}$ and $1.2 \mathrm{~K}$ at $4^{\circ}$ respectively. In addition, the temperature difference of $1^{\circ}$ 
and $4^{\circ}$ diverging-converging angle heat sink is $0.02 \mathrm{~K}$ and $1.2 \mathrm{~K}$ higher than that of $0^{\circ}$ respectively, and the change is not obvious. The reason for this change is that the increase of diverging-converging angle will make the fluid flow back in the channel and increase the local temperature. In addition, the maximum temperature and the temperature difference of $2^{\circ}$ diverging-converging angle heat sink increases by $0.63 \mathrm{~K}$ and $0.92 \mathrm{~K}$ respectively.

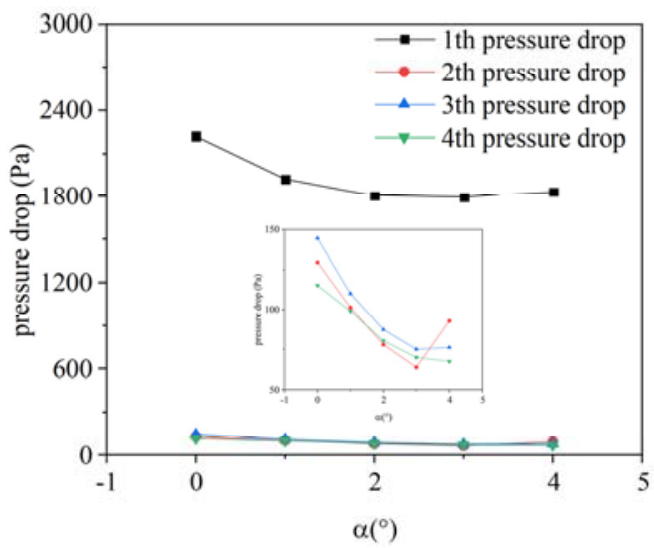

Figure 8. Effect of diverging-converging angle on pumping power and pressure drop of each branching level channel of tree-like network heat sink.

Figure 8 shows the pressure loss of each kth branching level channel in detail. The pressure loss of the 1 th branching level channel is large because the fluid is under developing section, while the pressure loss of other branching level is smaller. And the pressure drop of each branching level channel does not always decrease with the increase of diverging-converging angle. When the diverging-converging angle is $4^{\circ}$, the pressure drop of single channel increases instead, which is consistent with the change trend of total pressure drop with angle.

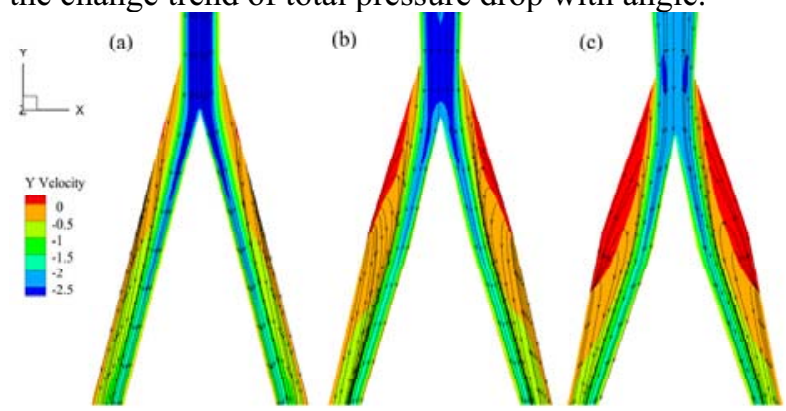

Figure 9 . The streamline of fluid colored by $\mathrm{Y}$ velocity in the 2th branching channel: (a). $0^{\circ}$ diverging-converging angle, (b). $2^{\circ}$ diverging-converging angle, (c). $4^{\circ}$ diverging-converging angle.

In order to analyze the reason of the pressure drop and temperature change with diverging-converging angle. Figure 9 compares the velocity and streamline of $0^{\circ}, 2^{\circ}$ and $4^{\circ}$ at 2 th branching level channel when mass flow rate is $0.0049 \mathrm{~kg} / \mathrm{s}$, and illustrates the details of the mixing and separation processes. The results show that when the fluid bifurcates from 1th channel to 2th channel, the streamline near the inner wall of the 2 th channel is more concentrated, and the velocity is larger. However, on the outer wall of the channel, there is a recirculation region, and the flow tends to deviate from the outer wall to the inner wall. In the $2^{\circ}$ diverging-converging angle tree-like network channel, due to the existence of $2^{\circ}$ divergingconverging angle, the degree of fluid separation at the outer wall is increased and becomes more obvious. Backflow exists in the outer wall of the channel. Compared with the tree-like network channel with $2^{\circ}$ angle and $4^{\circ}$ angle, it is found that with the increase of the angle, the degree of flow separation on the outer wall increases and the recirculation zone becomes larger. This is why the total pressure drop increases when the angle changes from $2^{\circ}$ to $4^{\circ}$.

\section{Conclusions}

The tree-like network heat sink with different divergingconverging angles is proposed, the flow and heat transfer performance is numerical studied. The results show that the total power of the tree-like network heat sink with $2^{\circ}$ diverging-converging angle is less than that with $0^{\circ}$ or $4^{\circ}$ diverging-converging angle. The reduction of the total pumping power ranges from $22 \% \sim 9 \%$, and decreases with increasing of flow rate. When the mass flow rate reaches $0.0066 \mathrm{~kg} / \mathrm{s}$, the wall temperature difference of the heat sink with $2^{\circ}$ diverging-converging angle increases by $0.49 \mathrm{~K} \sim 1.36 \mathrm{~K}$. It shows that the effect of the design on heat dissipation is not as obvious as on the pressure drop.

When the angle reaches $4^{\circ}$, the wall temperature difference increases by $1.2 \mathrm{~K}$. The reason is that the existence of diverging-converging angle will make the fluid flow back in the tree-like network channel, and increases the pressure loss and pumping power. Meanwhile, the fluid can't take away the heat in the recirculation zone, increasing local temperature.

The tree-like network heat sink with $2^{\circ}$ divergingconverging angle has a good performance in pressure drop without obvious influence on heat dissipation performance of heat sink. This provides a useful reference for drag reduction design of micro-channel heat sink.

\section{Acknowledgements}

The work was sponsored by the National Key R\&D Program of China (2018YFB1900602) and the National Natural Science Foundation of China (NSFC Grant No.11372302); Numerical calculations in present work were supported by Supercomputing Center of University of Science and Technology of China.

\section{References}

1. Y. Yan, H. Yan, L. Zhang, L. Li, J. Zhu, and Z. Zhang, Numerical investigation on combustion characteristics of methane/air in a micro-combustor with a regular triangular pyramid bluff body. International Journal of Hydrogen Energy, 43: 75817590,(2018).

2. D.B. Tuckerman and R.F.W. Pease, HighPerformance Heat Sinking for Vlsi. Electron Device Letters, 2: 126-129,(1981). 
3. G. Wang, Y. Gu, L. Zhao, J. Xuan, G. Zeng, Z. Tang, and Y. Sun, Experimental and numerical investigation of fractal-tree-like heat exchanger manufactured by 3D printing. Chemical Engineering Science, 195: 250-261,(2019).

4. H. Liu, B. Li, and J. Hong, Generating constructalconduction-networks for cooling discs at macro and micro scales. International Communications in Heat and Mass Transfer, 109,(2019).

5. X.-f. Yu, C.-p. Zhang, J.-t. Teng, S.-y. Huang, S.-p. Jin, Y.-f. Lian, C.-h. Cheng, T.-t. Xu, J.-c. Chu, Y.-J. Chang, D. Thanhtrung, and R. Greif, A study on the hydraulic and thermal characteristics in fractal treelike microchannels by numerical and experimental methods. International Journal of Heat and Mass Transfer, 55: 7499-7507,(2012).

6. B. Li, J. Hong, and L. Ge, Constructal design of internal cooling geometries in heat conduction system using the optimality of natural branching structures. International Journal of Thermal Sciences, 115: 16-28,(2017).

7. D.L. Jing and J. Song, Comparison on the hydraulic and thermal performances of two tree-like channel networks with different size constraints. International Journal of Heat and Mass Transfer, 130: 10701074,(2019).

8. X.-Q. Wang, A.S. Mujumdar, and C. Yap, Thermal characteristics of tree-shaped microchannel nets for cooling of a rectangular heat sink. International Journal of Thermal Sciences, 45: 1103-1112,(2006).

9. P. Xu, A.P. Sasmito, C. Li, and S. Qiu, Global and local transport properties of steady and unsteady flow in a symmetrical bronchial tree. International Journal of Heat and Mass Transfer, 97: 696704,(2016).

10. S. Xu, Y. Li, X. Hu, and L. Yang, Characteristics of heat transfer and fluid flow in a fractal multilayer silicon microchannel. International Communications in Heat and Mass Transfer, 71: 86-95,(2016).

11. M. Liang, Y. Gao, L. Luo, B. Xiao, M. Pang, and Z. Wang, A study on the permeability for the tree-like branching network with polygonal loops based on the fractal network of leaf vein. Chemical Engineering Science, 207: 911-928,(2019).

12. T.J. Miao, A.M. Chen, L.W. Zhang, and B.M. Yu, A novel fractal model for permeability of damaged tree-like branching networks. International Journal of Heat and Mass Transfer, 127: 278-285,(2018).

13. D. Jing, S. Song, and L. He, Reexamination of Murray's law for tree-like rectangular microchannel network with constant channel height. International Journal of Heat and Mass Transfer, 128: 13441350,(2019).

14. H.-1. Liu, Y.-q. Shao, Z.-t. Chen, and Z.-1. Xie, Heat transfer and flow performance of a novel $T$ type heat sink with GaInSn coolant. International Journal of Thermal Sciences, 144: 129-146,(2019).

15. S. Xu, W. Wang, K. Fang, and C.-N. Wong, Heat transfer performance of a fractal silicon microchannel heat sink subjected to pulsation flow. International Journal of Heat and Mass Transfer, 81: 33-40,(2015).

16. S. Chester, C. Meneveau, and M.B. Parlange, Modeling turbulent flow over fractal trees with renormalized numerical simulation. J Comput Phys, 225: 427-448,(2007).

17. V.S. Duryodhan, S.G. Singh, and A. Agrawal, Liquid flow through converging microchannels and a comparison with diverging microchannels. Journal of Micromechanics and Microengineering, 24,(2014).

18. S.-S. Hsieh and J.-H. Liou, DNA molecule dynamics in converging-diverging microchannels. Biotechnol Appl Biochem, 52: 29-40,(2009).

19. X.C. Xuan and D.Q. Li, Particle motions in lowReynolds number pressure-driven flows through converging-diverging microchannels. Journal of Micromechanics and Microengineering, 16: 6269,(2006).

20. J. Samuelsson, O. Tammisola, and M.P. Juniper, Breaking axi-symmetry in stenotic flow lowers the critical transition Reynolds number. Physics of Fluids, 27,(2015).

21. M.D. Griffith, T. Leweke, M.C. Thompson, and K. Hourigan, Effect of small asymmetries on axisymmetric stenotic flow. Journal of Fluid Mechanics, 721,(2013).

22. M. Sarkar, S.B. Paramane, and A. Sharma, Periodically Fully Developed Heat and Fluid Flow Characteristics in a Furrowed Wavy Channel. Heat Transfer Engineering, 38: 278-288,(2017).

23. J. Vetel, A. Garon, D. Pelletier, and M.I. Farinas, Asymmetry and transition to turbulence in a smooth axisymmetric constriction. Journal of Fluid Mechanics, 607: 351-386,(2008).

24. X.Q. Wang, A.S. Mujumdar, and C. Yap, Numerical analysis of blockage and optimization of heat transfer performance of fractal-like microchannel nets. Journal of Electronic Packaging, 128: 3845,(2006).

25. Y. Yan, H. Yan, S. Yin, L. Zhang, and L. Li, Single/multi-objective optimizations on hydraulic and thermal management in micro-channel heat sink with bionic Y-shaped fractal network by genetic algorithm coupled with numerical simulation. International Journal of Heat and Mass Transfer, 129: 468-479,(2019).

26. X.L. Xie, Z.J. Liu, Y.L. He, and W.Q. Tao, Numerical study of laminar heat transfer and pressure drop characteristics in a water-cooled minichannel heat sink. Applied Thermal Engineering, 29: 64-74,(2009). 